\title{
Control of Resonant Acoustic Sound Fields by Electrical Shunting of a Loudspeaker
}

\author{
Andrew J. Fleming, Member, IEEE, Dominik Niederberger, S. O. Reza Moheimani, Senior Member, IEEE, and \\ Manfred Morari, Fellow, IEEE
}

\begin{abstract}
Low-frequency reverberant sound fields are usually suppressed by means of either adaptive feedforward control or Helmholtz resonator. Feedforward systems utilize a noise reference signal, error microphone, and loudspeaker to cancel sound propagating in one direction. Due to the requirement for multiple transducers and a powerful digital signal processor, feedforward systems are the most complex and expensive option for acoustic noise reduction. Helmholtz resonators, comprising auxiliary coupled acoustic chambers, are a popular passive technique for the control of dominant acoustic modes. Although lightly damped acoustic modes can be heavily attenuated, the resonators are difficult to tune and require impractically large cavity volumes at frequencies below $200 \mathrm{~Hz}$. This paper introduces a new technique for the control of low-frequency reverberant sound fields. By connecting an electrical impedance to the terminals of an acoustic loudspeaker, the mechanical dynamics, and hence, acoustic response can be made to emulate a sealed acoustic resonator. No microphone or velocity measurement is required. In some cases, the required electrical circuit is simply the parallel connection of a capacitor and resistor. With the addition of a single pressure microphone, a technique for online circuit adaptation is proposed. Experimental application to a closed acoustic duct results in 14-dB pressure attenuation of a single acoustic mode. Active impedances can be designed by viewing the system model from a feedback control perspective. The resulting electrical impedances, although not passive, are experimentally shown to attenuate four acoustic modes by up to $10 \mathrm{~dB}$.
\end{abstract}

Index Terms-Acoustic impedance, acoustic noise, loudspeakers, optimal control, passive circuits, shunt damping.

\section{INTRODUCTION}

$\mathbf{S}$ INCE the original work of Leug [1] and Olson and May [2], the problem of low-frequency acoustic noise attenuation has been studied throughout the industrial and academic domains. High-frequency acoustic noise (greater than $500 \mathrm{~Hz}$ )

Manuscript received April 12, 2005; revised May 5, 2006. Manuscript received in final form October 25, 2006. Recommended by Associate Editor I. Fialho. This work was supported in part by the Australian Research Council, by the Centre for Complex Dynamic Systems and Control, by the Swiss Federal Institute of Technology Zurich, and by the Swiss Federal Laboratory for Material Testing and Research Dubendorf.

A. J. Fleming and S. O. R. Moheimani are with the School of Electrical Engineering and Computer Science, University of Newcastle, Callaghan, NSW 2308, Australia (e-mail: andrew.fleming@newcastle.edu.au; reza.moheimani@newcastle.edu.au).

D. Niederberger is with the SENSIRION-The Sensor Company, CH-8712 Staefa, ZH, Switzerland (e-mail: dominik.niederberger@ sensirion.com).

M. Morari is with the Institut für Automatik, ETH-Eidgenossische Technische Hochschule, CH-8092 Zürich, Switzerland (e-mail: morari@ control.ee. ethz.ch).

Color versions of Figs. 3, 4, and 10 are available online at http://ieeexplore. ieee.org.

Digital Object Identifier 10.1109/TCST.2006.890276 is generally addressed with a combination of porous damping materials [3], Helmholtz resonators [4], [5], and mufflers [5]. Unfortunately such technologies do not offer acceptable absorbance at lower frequencies. Porous damping materials rely on the viscous damping of fluid flow over a surface. As particle velocity is proportional to frequency, impractical volumes of material are required at frequencies below $500 \mathrm{~Hz}$. Helmholtz resonators, the acoustic equivalent of a mechanical-tuned mass absorber, provide excellent attenuation of highly resonant acoustic modes but require restrictively large cavity volumes at frequencies below $200 \mathrm{~Hz}$. The inadequacy of traditional passive damping treatments has motivated a diverse literature on the active control of low-frequency reverberant noise.

As shown in Fig. 1, the field of nontraditional acoustic noise control can be grouped roughly into five categories: 1) passive baffles and compliant panels; 2) helmholtz resonators; 3) feedforward noise control; 4) feedback noise control; and 5) impedance-based. A commentary follows on each of the classifications.

\section{A. Passive Absorbers}

Helmholtz resonators [4] have been used extensively for the attenuation of highly resonant acoustic modes. A secondary acoustic cavity, as shown in Fig. 1(a), is connected to the primary enclosure through a small port. The cavity [6] and port dimensions are tuned to damp a single acoustic mode. As many resonators are required as there are modes to control. Although Helmholtz resonators can provide greater than $20 \mathrm{~dB}$ attenuation of lightly damped acoustic modes, they are also bulky and require experimental tuning. Practical implementations have included mechanisms for adapting the cavity volume or port width online [7], [8]. Hybrid systems combining passive resonators with active feedback control, have also been proposed as a method for increasing the absorption bandwidth [9].

Compliant panels, another passive technique, have been studied theoretically [10], [11], and experimentally [12], [13] A flexible membrane or panel, mounted into the wall of an enclosure, can reduce acoustic response by altering the mechanical boundary conditions. Although compliant panels are simple to construct, flexible membranes are unsuitable for static pressure loads, and are difficult to optimize both theoretically and experimentally.

\section{B. Feedforward}

Since the first application of digital signal processors in active noise control [14], feedforward techniques, as shown in 


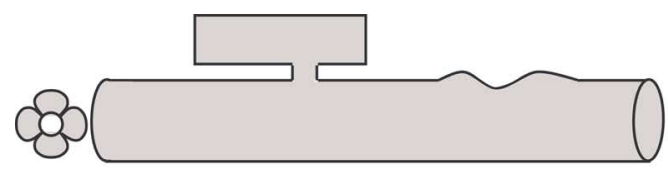

(a) Helmholtz Resonator and Compliant Panel

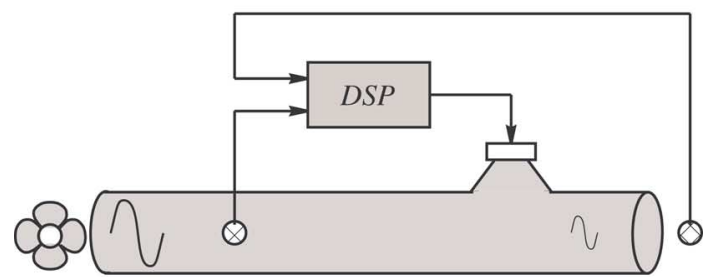

(b) Feedforward Control

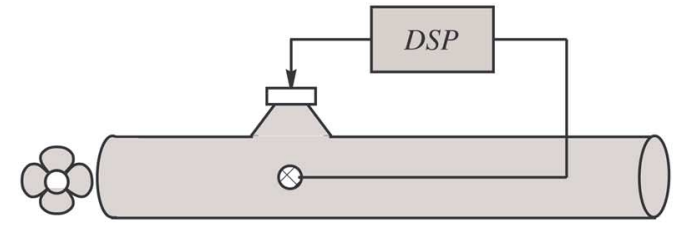

(c) Feedback Control

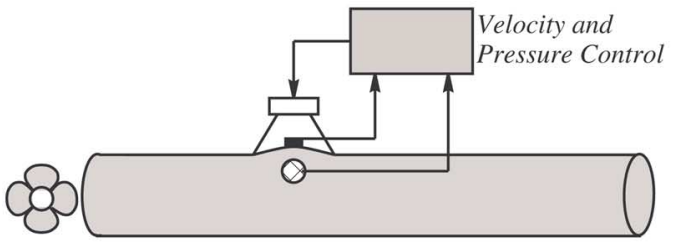

(d) Impedance Based

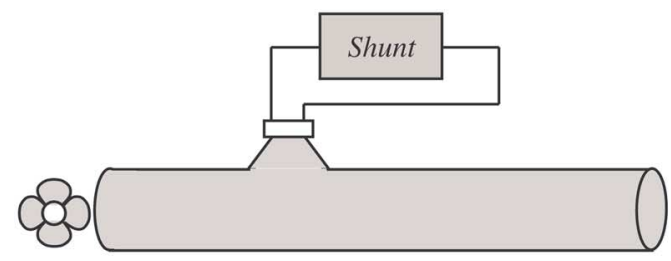

(e) Shunt Control

Fig. 1. Approaches to acoustic noise control. (a) Passive absorbers like Helmholtz resonators or flexible membranes. (b) Feedforward control by using a reference signal that is filtered and applied "downstream." (c) Feedback control by regulating the measured pressure. (d) Impedance-based control by passively or actively manipulating the acoustic impedance. (e) Electrical shunt control as presented in this paper.

Fig. 1(b), have been the dominant technology. Techniques categorized as feedforward are distinguished by the use of a reference signal, filtered and applied "downstream" to arrest disturbance propagation. As open-loop control systems, the performance of a feedforward system is extremely sensitive to plant or filter variation. Although some fixed filter designs have been presented, the feedforward filter is generally tuned adaptively with the aid of an error microphone. For a full history and introduction to active noise control, the reader is referred to a tutorial by Kuo and Morgan [15], [17] and the texts by Nelson and Elliot [16].

The use of adaptive filters significantly simplifies the application of active noise control as no model of the acoustic system is required. This is significant since reverberant acoustic systems are not only difficult to identify, they can vary dramatically throughout service due to the movement of internal objects or changes in boundary conditions.
Although adaptive filters are simple in concept, they require significant processing resources to provide adequate real-time performance. The additional need for error microphones and reference signals makes adaptive feedforward techniques the most equipment intensive.

Practical implementation requires filters to account for the cancellation of speaker feedback to the reference signal, transducer dynamics, and performance localization. Such additional requirements have not hindered the wide spread application of feedforward noise control in applications varying from air conditioning noise control [18], to multichannel attenuation of aircraft cabin noise [19].

\section{Feedback}

Feedback control of acoustic noise, using a loudspeaker to regulate the measured pressure, has been studied vigorously in the control and acoustics literature since the early 1990s. Although the work was well motivated, namely to eliminate the need for a feedforward reference sensor, subsequent theoretical and laboratory works have been criticized for their impracticality [20]. Similar to the first active absorber introduced in 1953 [2], early active feedback methods considered only the regulation of pressure at a single microphone location. Although a number of works appeared stressing the need for spatial, energybased, or dissipative control [21]-[23], a plethora of control design methodologies has been applied to the ill-posed singleoutput 1-D duct problem.

The majority of studies in acoustic active feedback neglect the spatially distributed nature of acoustic enclosures, and consequently, offer extremely poor or detrimental performance at a distance from the error microphone. Spatial models, as required to ensure global performance are, unfortunately, extremely difficult to obtain. Analytic modeling of even the simplest acoustic system is a challenging and often unproductive task. System identification, yielding little spatial information is often employed to procure the necessary model for control design.

One of the foremost, and largely neglected, reasons for inaccuracy in analytic modeling is the presence of passive mechanical loudspeaker dynamics. Although some authors have gone to the extent of identifying an actuator transfer function, measured from an applied voltage to the baffle velocity in free space [24], such a model cannot describe the acoustic coupling between the loudspeaker and enclosure. From a feedback control perspective, Clark and Cole were first to realize the importance of passive loudspeaker dynamics. In [23], simulations of collocated direct pressure feedback with an ideal velocity source were shown to dissipate acoustic energy from an enclosed sound field. This work was demonstrated experimentally in [25] using a feedback compensated loudspeaker. Details of the original speaker compensation can be found in [26], a more recent observer-based design can be found in [27]. Although it may be difficult to adequately compensate the speaker and achieve perfect sensor collocation, direct rate pressure-velocity feedback has been cited as the most applicable feedback technique. An extension including pressure self-sensing was presented in [28].

In an early work, before implementing the constant volume velocity source, Clark and Frampton [29] present the acousticmechanical coupling as a feedback diagram. The mechanical 
dynamics are likened to a passive pressure feedback controller. A similar perspective is used in this work to artificially improve the dissipation of a passive loudspeaker.

\section{Impedance-Based}

Impedance-based control involves the passive or active manipulation of acoustic impedance within an acoustic enclosure. The foremost motivations for impedance-based control are reduced complexity, robustness, intuitive acoustic design, and global performance. Active impedance control was first demonstrated by Guicking and Karcher [30], where a loudspeaker baffle was used to implement an absorbing acoustic impedance at the end of a duct. A velocity controlled loudspeaker and microphone was used to directly implement the acoustic impedance. Guicking and Lorentz [31] also demonstrated the use of a loudspeaker to increase the performance of a porous absorber. A loudspeaker and microphone were used to simulate the impedance of a traditional quarter-wavelength cavity placed behind the porous material to increase absorption. Porous materials combined with active "acoustic virtual earths" are simple to implement, do not require loudspeaker acceleration or velocity control, and provide reasonable low-frequency absorption. An extension of these techniques to 3-D enclosures with arbitrary incidence angle and spatial response was presented in [32] and [33].

The main disadvantage of active impedance control is the difficulty in achieving tight velocity or pressure feedback control of the loudspeaker. If the added complexity is tolerable and the disturbance measurable, adaptive feedforward is an option for implementing the impedance control [34]. Active impedance control also suffers from the necessity for a high-quality collocated pressure measurement. Motion or self-sensing pressure measurement is not considered to be of sufficient fidelity. A theoretical study using only a secondary speaker coil has been presented for active impedance control [35]. The author predicts practical difficulties due to high gains and the need for a differentiator.

Recognizing the need for simplicity and reliability in critical applications such as launch vehicle acoustic control, Kemp and Clark [36] present a novel passive acoustic absorber. The short-circuit coil resistance and spring constant of a mechanical loudspeaker are optimized to damp a single acoustic resonance. In the absence of an ideal speaker, an accelerometer and model reference controller are used to tune the dynamics of the nearest suitable candidate. In their practical implementation, the acoustic response of the system is identified, then used to calculate the optimal loudspeaker response. Such a technique is susceptible to modeling errors in the identified acoustic system and loudspeaker reference model.

\section{E. Electrical Shunt Control}

In this paper, we present a new technique for the attenuation of reverberant sound fields. The goal is to globally reduce acoustic response without the need for either a precise plant model, collocated pressure sensor, or constant volume velocity source. By identifying the interaction between sound-field, mechanical speaker, and electromagnetic transducer, a simple electrical impedance can be designed, which when connected

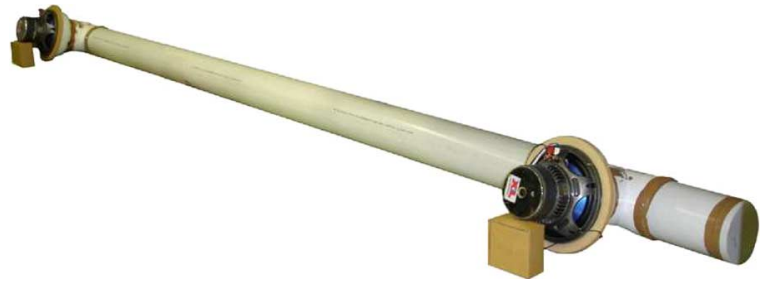

Fig. 2. Experimental duct with two transversely mounted loudspeakers.

to a speaker coil, improves the dissipation of acoustic energy. The designed electrical impedance that effectively renders the speaker as an acoustic resonator does not require a model for design and can be tuned experimentally or adaptively. Under certain circumstances, the electrical impedance can be simplified to a passive resistor and capacitor.

For increased performance, if a system model is available, an active electrical impedance can be designed that augments the natural damping of an acoustic system.

In Section II, the electro-mechanical-acoustic coupling is described. An electrical equivalent model is also presented that facilitates intuitive impedance design. In Sections III and IV, passive and active impedance designs are applied to an experimental duct system. The conclusion appears in Section V.

\section{SySTEM DYNAMICS}

The majority of techniques for acoustic noise control are targeted at a specific problem scenario. In this paper, our objective is to reduce pressure variation within a sealed duct in response to a planar velocity disturbance. The experimental apparatus, comprising a PVC pipe, with two transversely mounted speaker ports is shown in Fig. 2. Dimensions and the location of pressure, velocity, and electrical measurements can be found in Fig. 3.

A velocity disturbance is introduced artificially using Speaker 1. By exciting the speaker with a voltage, the applied velocity disturbance is measured directly using a Polytec laser vibrometer (PSV300). By considering the disturbance speaker velocity as an input, rather than the voltage or current, the speaker appears as a pure velocity source, i.e., a rigid wall, and does not interact with the duct dynamics. The two pressure measurements $p_{1}$ and $p_{2}$ are acquired using $\mathrm{B} \& \mathrm{~K} 4935$ array microphones with a Nexus preamplifier. While $p_{1}$ is used for performance evaluation, $p_{2}$ is required for analysis and control design purposes. The performance measurement is located at the opposite end of the duct to illustrate the global performance of the proposed controllers. The velocity of the control speaker, Speaker 2, is also measured using the vibrometer. An internal view of the control speaker in Fig. 4 shows the orientation of the baffle pressure microphone. When the speaker is mounted onto the duct, the baffle velocity is measured through the rear air vent.

The experimental apparatus was chosen to represent a simple 1-D reverberant noise control problem. Although the apparatus most closely resembles an air conditioning duct, multidimensional extension to reverberant room noise is possible. Another system closely resembling the experimental apparatus is a launch vehicle payload enclosure. End-mounted speakers have 


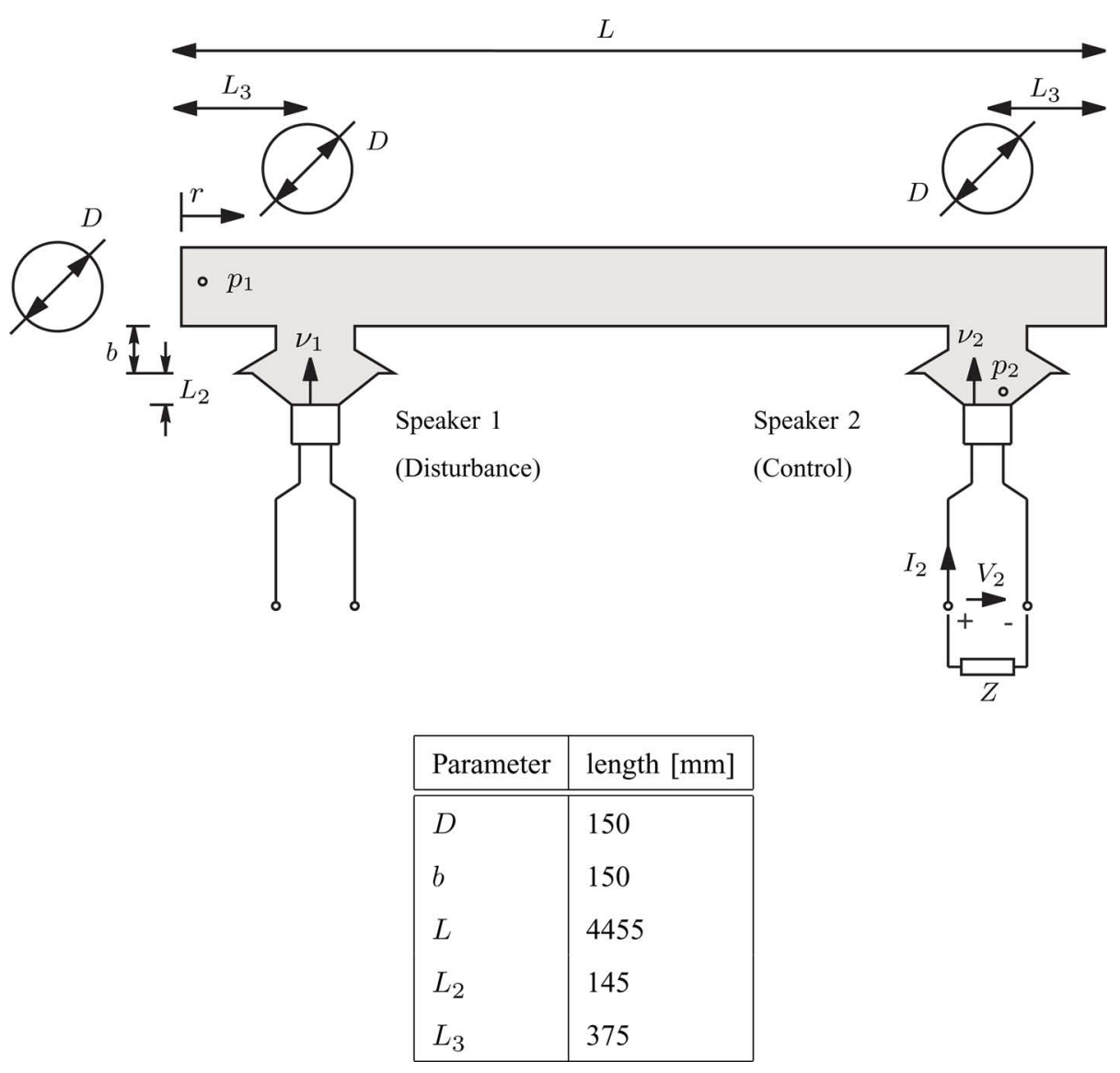

Fig. 3. Acoustic duct geometry and dimensions.

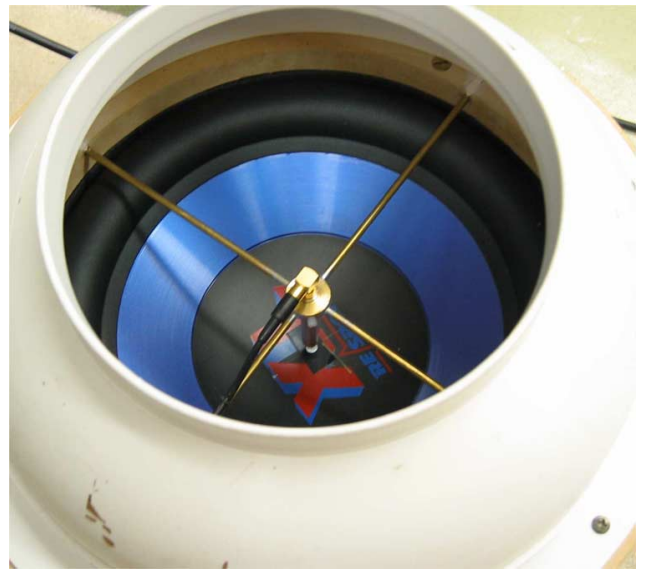

Fig. 4. Internal view of the control speaker with baffle pressure microphone.

been used with acoustic impedance-based techniques for the mitigation of acoustic pressure forces on sensitive payloads [36].

In the following sections, a dynamic model of the duct system is derived progressively. We begin by considering an ideal acoustic system, i.e., a hard-walled enclosure disturbed by a perfect velocity source. The passive mechanical dynamics of a control loudspeaker are then augmented to the ideal acoustic system. Finally, the electromagnetic model is also included to relate the coil voltage and current to the duct pressure and velocity. Many works in acoustic noise control simply neglect passive dynamics by modeling each loudspeaker as a velocity source. Although such works present analytic models, an extremely poor correspondence to experimental data is often observed. It is likely that a considerable portion of this disagreement can be attributed to unmodeled passive loudspeaker dynamics.

Other authors have also considered nonideal acoustic systems, for example, systems with "soft" walls, nontrivial end impedances [37], and passive speaker dynamics [29], [37]. In this paper, the main objective is not to develop an exact model of the physical system, but rather to reveal the coupling and interaction between each domain. By illustrating the coupling in a graphical manner, simple impedance designs can be derived by inspection and experimental tuning.

\section{A. Acoustic Dynamics}

The governing equations relevant to the modeling of an acoustic enclosure are the fundamental equations of fluid mechanics: mass conservation, equation of state, and Euler's equation of motion. Linearization is possible in cases of small pressure perturbation and zero-mean fluid velocity. The following wave equation for the acoustic duct shown in Fig. 3 (without the control Speaker 2) can be derived by combining 


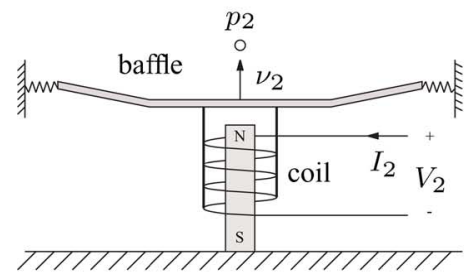

(a)

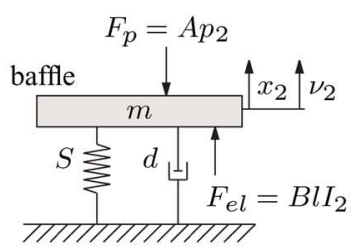

(b)

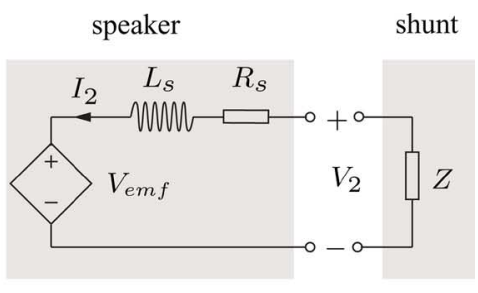

(c)

Fig. 5. Loudspeaker specification and modeling. (a) Physical sketch. (b) Mechanical equivalent diagram. (c) Electrical equivalent diagram.

the fundamental equations and solving for the fluid pressure [16]

$$
\frac{\partial^{2} p(r, t)}{\partial r^{2}}-\frac{1}{c^{2}} \frac{\partial^{2} p(r, t)}{\partial t^{2}}=\rho_{0} \frac{d \nu_{1}(t)}{d t} \delta\left(r-L_{3}\right)
$$

subject to the closed-end boundary conditions

$$
\nu(0, t)=\nu(L, t)=0
$$

where $p(r, t)$ is the sound pressure variation measured $r$ (meters) from the duct end, $\nu_{1}(t)$ is the forced velocity $L_{3}$ (meters) from the duct end, $\rho_{0}$ is the ambient density, and $c$ is the speed of sound.

In the design and analysis of acoustic shunt impedances, the transfer function relating pressure to velocity at the control speaker baffle $G_{p_{2} \nu_{2}}(s)$ is of primary interest. A number of techniques are available in the literature for deriving closed-form solutions to (1). Examples considering side-mounted speakers can be found in references [22], [24], and [38].

The majority of practical acoustic systems contain properties difficult to model analytically, e.g., nonideal boundary conditions, irregular geometries, etc. It may be impossible to find a closed-form solution to the acoustic transfer function. In such cases, finite element analysis or system identification may present a viable alternative. The latter technique is applied in Section IV to provide a lumped model of the electromechanical and acoustic systems.

\section{B. Including Loudspeaker Mechanical Dynamics}

A physical sketch and the equivalent mechanical diagram of an acoustic loudspeaker is illustrated in Fig. 5(a) and (b). The mass, spring, and damping components correspond to the baffle weight, suspension stiffness, and suspension damping, respectively. In this section, the unshunted system is considered, i.e., when $I_{2}=0$ which implies $F_{\text {el }}=0$.

The response of the speaker can be expressed as a simple mass-spring-damper system

$$
\ddot{x}_{2}=\frac{1}{m}\left(-F_{p}-d \dot{x}_{2}-S x_{2}\right)
$$

where $x_{2}$ denotes the inward baffle displacement, $F_{p}$ is the acoustic force related to the pressure $p_{2}$, and $m, S$, and $d$ are the equivalent mass, stiffness, and damping coefficients. In order to couple the loudspeaker and acoustic system, we require a transfer function $G_{\nu f}(s)$ relating the total applied force (in the

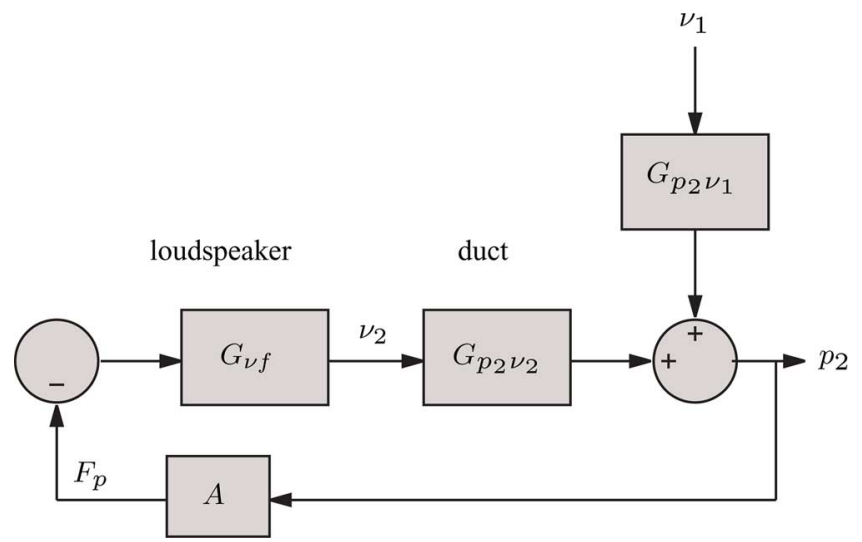

Fig. 6. Block diagram of the composite acoustic and passive loudspeaker dynamics.

direction opposite to $F_{p}$ ) to the baffle velocity $\nu_{2}$. From (3), $G_{\nu f}(s)$ can be written in the frequency domain as

$$
G_{\nu f}(s)=\frac{\nu_{2}(s)}{-F_{p}(s)}=\frac{s}{s^{2} m+d s+S} .
$$

By noting that the acoustic pressure develops a force proportional to the baffle surface area $F_{p}=A p_{2}$, and that the resulting velocity $\nu_{2}$ excites the acoustic system $G_{p_{2} \nu_{2}}(s)$, the two systems can be coupled. Fig. 6 clearly illustrates the equivalence between a pressure feedback controller and the passive loudspeaker dynamics. The disturbance transfer function, measured from $\nu_{1}$ to $p_{2}$ is modified from the open-loop response $G_{p_{2} \nu_{1}}(s)$ to

$$
\frac{p_{2}(s)}{\nu_{1}(s)}=\frac{G_{p_{2} \nu_{1}}(s)}{1+C(s) G_{p_{2} \nu_{2}}(s)}
$$

where the equivalent feedback controller $C(s)$ is

$$
C(s)=G_{\nu f}(s) A \text {. }
$$

Given that the loudspeaker acts to control the acoustic system, it is pertinent to identify and optimize the desirable characteristics during selection. The most obvious technique for increasing speaker influence is to increase the baffle area, as this directly affects the gain in the pressure feedback loop. The magnitude of the transfer function $G_{\nu f}(s)$ is also critical. Due to the gain 


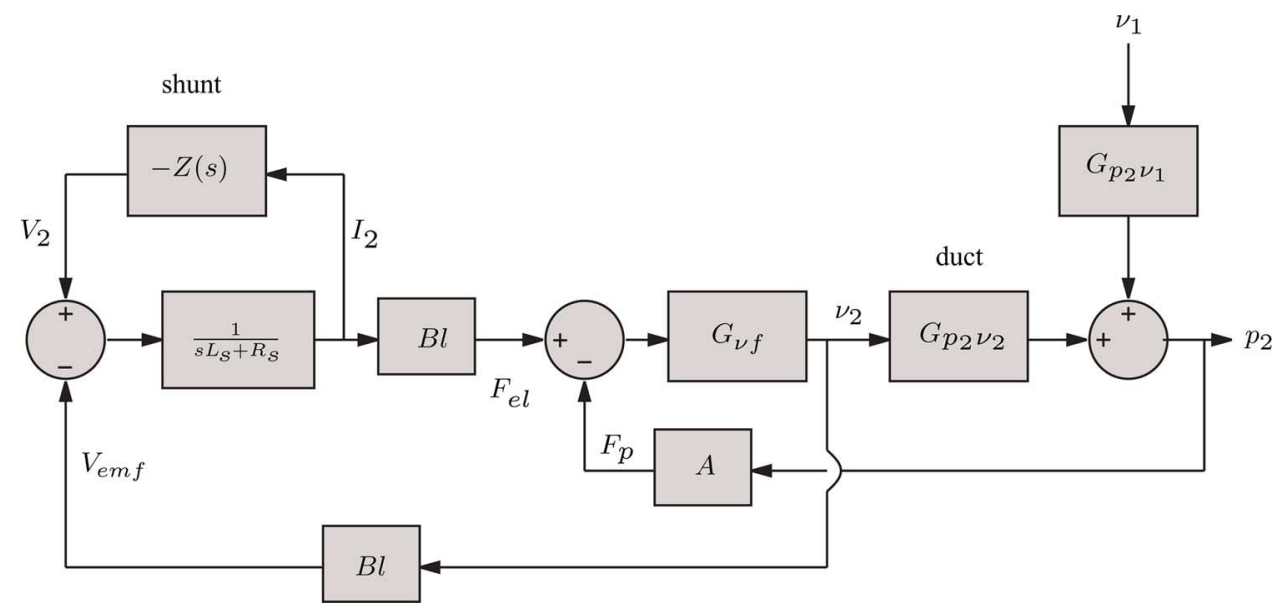

Fig. 7. Block diagram of the composite acoustic, passive loudspeaker, and electromagnetic dynamics.

roll-off of $20 \mathrm{~dB}$ per decade at frequencies greater than the mechanical cutoff, only low-frequency acoustic modes will be passively attenuated.

If a single dominant acoustic mode is the primary concern, a speaker with a lightly damped mechanical resonance, near in frequency, will achieve the greatest damping. The baffle resonance frequency can be altered by adding mass to the speaker cone. This should be done conservatively, as reducing the mechanical bandwidth of the speaker will also reduce the effective bandwidth over which the acoustic system can be controlled. Considering a single mode, if the frequency of mechanical and acoustic resonance is properly matched, the damping of the controller can be altered by shunting the coil with a resistor. As discussed fully in the following sections, if the inductive coil reactance is small, a resistive shunt will add mechanical damping.

\section{Including Electromagnetic Dynamics}

As shown in Fig. 5(c), the electromagnetic speaker dynamics can be modeled as a velocity dependent voltage source $V_{\text {emf }}$, in series with a resistor $R_{s}$, and inductor $L_{s}$. The induced voltage is related to the velocity by

$$
V_{\mathrm{emf}}=B l \nu_{2}
$$

where $B$ is the magnetic flux density and $l$ is the conductor length. If the speaker is short circuited, the orientation of the induced voltage, and hence induced current, hinders the application of an external velocity.

The force developed due to a current $I_{2}$ is equal to

$$
F_{\mathrm{el}}=B l I_{2}
$$

where $F_{\mathrm{el}}$ acts in the opposite direction to $F_{p}$ and hence adds to the total force applied to the speaker, i.e.,

$$
\nu_{2}(s)=G_{\nu f}(s)\left[F_{\mathrm{el}}(s)-F_{p}(s)\right] .
$$

If an electrical impedance $Z(s)$ is connected to the terminals of the speaker coil, the total voltage drop across the speaker impedance $s L_{s}+R_{s}$ is equal to the difference of the voltages across the speaker $V_{2}$, and $V_{\text {emf }}$, i.e., the current flowing through the coil can be written as

$$
I_{2}=\left(V_{2}-V_{\mathrm{emf}}\right) \frac{1}{s L_{s}+R_{s}} .
$$

These relations are shown graphically in Fig. 7. The electromagnetic dynamics introduce a further feedback loop around the velocity $\nu_{2}$. In the special case where the loudspeaker is short circuited, i.e., when $V_{2}=0$, and at frequencies below the cutoff of the filter $1 /\left(s L_{s}+R_{s}\right)$, the coil acts as an electrodynamic brake. Physical damping is added to the mechanical speaker dynamics. Too much mechanical damping will reduce the acoustic performance of the speaker. A properly chosen resistance can be used to optimize the damping and passive acoustic mitigation.

Physical coupling between the electrical and acoustic domain is limited in each direction by the factor $B l$. Good quality speakers with rare earth magnets and dense, low-impedance windings will provide the best shunt circuit performance.

\section{Helmholtz Resonators}

Helmholtz resonators can reduce the response of undesirable acoustic modes by effecting high absorption over a narrow frequency range. As shown in Fig. 8(a), a Helmholtz resonator comprises a cavity volume $V$ coupled to the host sound field through a short tube of cross section $A$ and length $l$. In this figure, $\nu$ and $p$ represent the air particle velocity and pressure at the resonator opening.

Due to the physical similarity to a lumped single-degree-offreedom system, Helmholtz resonators can be equivalently represented as an electrical network. A diagrammatic representation of the equivalent acoustic, mechanical, and electrical systems appears in Fig. 8(a)-(c), where $c$ is the speed of sound, $A$ the cross section, $l$ the length of the tube, $\rho$ the air density, and $V$ the cavity volume. The stiffness $c^{2} \rho / V$ is a function of the enclosed air volume $V$, while the mass $\rho A\left(l+l_{\text {corr }}\right)=$ $\rho A l_{\text {eq }}$ corresponds to the accelerated air in the tube, where $l_{\text {corr }}$ is a correction factor accounting for additional air-mass at the tube opening. The correction factor is typically taken as $l_{\text {corr }}=$ $0.8 R$, where $R$ is the radius of the tube. 


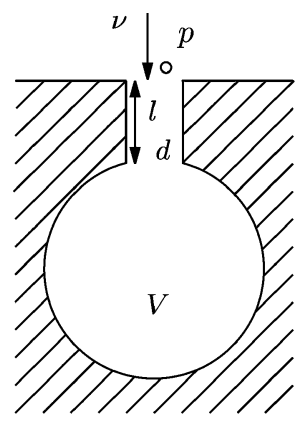

(a)

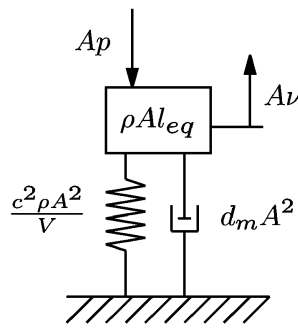

(b)

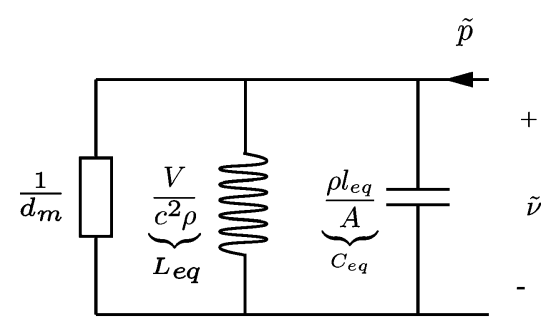

(c)

Fig. 8. Specification and model of a Helmholtz resonator. (a) Physical sketch. (b) Equivalent mechanical model. (c) Equivalent electrical model.

By inserting damping materials in the tube, where air particle velocity is maximum, the damping $d$ of the resonance can be increased. Note that the corresponding electrical parameters in Fig. 8(c) are scaled by the factor $1 / A^{2}$, this arises from the conversion $\tilde{p}=A p$ and $\tilde{v}=A v$. The resonance frequency of the Helmholtz resonator without damping is [6]

$$
\omega_{\mathrm{res}}=c \sqrt{\frac{A}{V\left(l+l_{\mathrm{corr}}\right)}}=c \sqrt{\frac{A}{V l_{\mathrm{eq}}}}=\frac{1}{\sqrt{L_{\mathrm{eq}} C_{\mathrm{eq}}}}
$$

where $L_{\mathrm{eq}}=V / c^{2} \rho$ and $C_{\mathrm{eq}}=\rho l_{\mathrm{eq}} / A$.

\section{E. Electrical Equivalent System}

To aid in the understanding of the composite electrical, mechanical, and acoustic systems, as shown in Fig. 7, it is helpful to cast each subsystem in the same physical domain. Given that our objective is to design a suitable shunt impedance, the choice of electrical domain permits a significant simplification of the complicated interactions shown in Fig. 7.

The equivalent electrical network of the composite speakerenclosure model is shown in Fig. 9(a). The mechanical part of the speaker is modeled as a baffle mass $m$, a stiffness $S$, and mechanical damping $d$. The baffle is excited by the electrical force $F_{\text {el }}$ proportional to the current $I_{2}$. The relationship $F_{\text {el }}=$ $B l I_{2}$ is represented by a transformer. Analogously, the baffle acoustic force $F_{p}=A p_{2}$, is modeled by a gyrator.

In Fig. 9(a), only a single acoustic mode is considered and the volume air velocity at the baffle is denoted by $\nu_{v}=A \nu_{2}$. The electrical part of the loudspeaker is modeled by the coil resistance $R_{s}$, inductance $L_{s}$, and induced voltage $V_{\text {emf }}=B l \nu_{2}$.

By eliminating the gyrators and transformers using dual network elements and converting the values with $A^{2}$ and $(B l)^{2}$, respectively, one obtains the simplified circuit shown in Fig. 9(b). The corresponding mechanical part of the speaker performs like a Helmholtz resonator with resonance frequency $\omega_{\text {res }}=\sqrt{\hat{S} / \hat{m}}$ and damping $\hat{d}$. It is shown in the following, that these parameters can be modified through the application of a suitable electrical shunt impedance $Z(s)$.

\section{Passive Shunt Circuit Design}

In analogy to the field of piezoelectric shunt damping [39], [40], where an electrical circuit is shunted to the terminals of a piezoelectric transducer, a network connected to the terminals of a loudspeaker can be designed to moderate the response of a coupled acoustic enclosure. In this section, the design of passive shunt circuits is discussed.

Based on the electrical equivalent model introduced in Section II-E, one observes that an enclosed speaker emulates the acoustic response of a Helmholtz resonator. If the properties of this virtual Helmholtz resonator can be adjusted, the speaker can be employed to attenuate a highly resonant acoustic mode in the same fashion as a physical Helmholtz resonator. In the following, shunt circuit topologies are presented that allow the parameters of the virtual Helmholtz resonator to be modified.

\section{A. $R$ Shunts}

At low frequencies where $\omega \ll R_{s} / L_{s}$, the influence of the inductor $L_{s}$ can be neglected. In this case, the Helmholtz damping $\hat{d}$ can be increased by connecting a resistor $R$ to the terminals of the speaker. As observed in Fig. 9(b), the resistor $R$ in addition to the coil resistor $R_{s}$, appears in parallel to the mechanical damping $\hat{d}$. The total damping of the virtual Helmholtz resonator is then

$$
\hat{d}_{\mathrm{tot}}=\hat{d}+\frac{1}{R+R_{s}} .
$$

Note that the total damping is restricted in range between $\hat{d}$ and $\hat{d}+1 / R_{s}$. A speaker with low $\hat{d}$ and $R_{s}$ will provide the greatest tuning range.

The experimental application of a resistive shunt to the duct apparatus is shown in Fig. 10(a). For small values of $R$, the Helmholtz damping is large, thus, no influence on the duct system can be observed. For larger values of $R$, the resonator becomes more lightly damped until an antiresonance appears at $40 \mathrm{~Hz}$ with new sidelobes at 30 and $50 \mathrm{~Hz}$. As the Helmholtz frequency is improperly tuned, this system is not effective at suppressing noise.

\section{B. $C / / R$ Shunts}

In the low frequency regime, i.e., where $\omega \ll R_{s} / L_{s}$, a parallel $C / / R$ shunt circuit provides authority over the damping and resonance frequency of a virtual Helmholtz resonator. The addition of a parallel capacitor $C_{p}$ effects an increase in the parameter $1 / \hat{S}$. Thus, the Helmhotz resonance frequency can be reduced. 


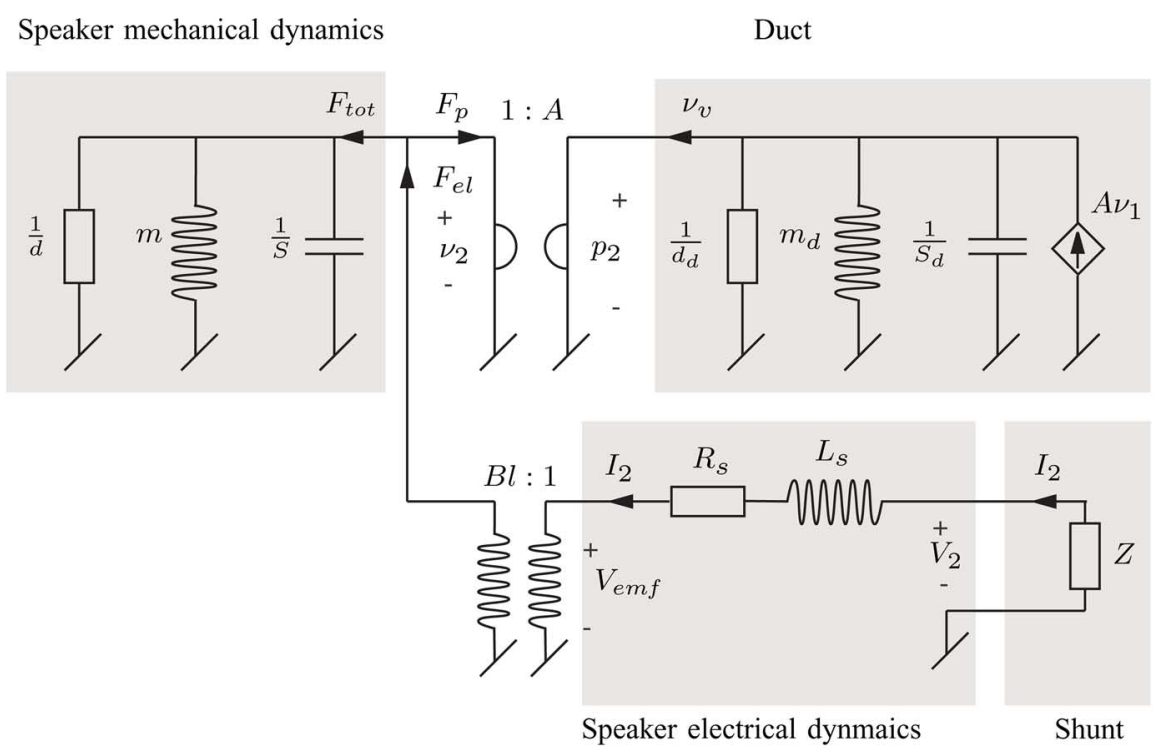

(a)

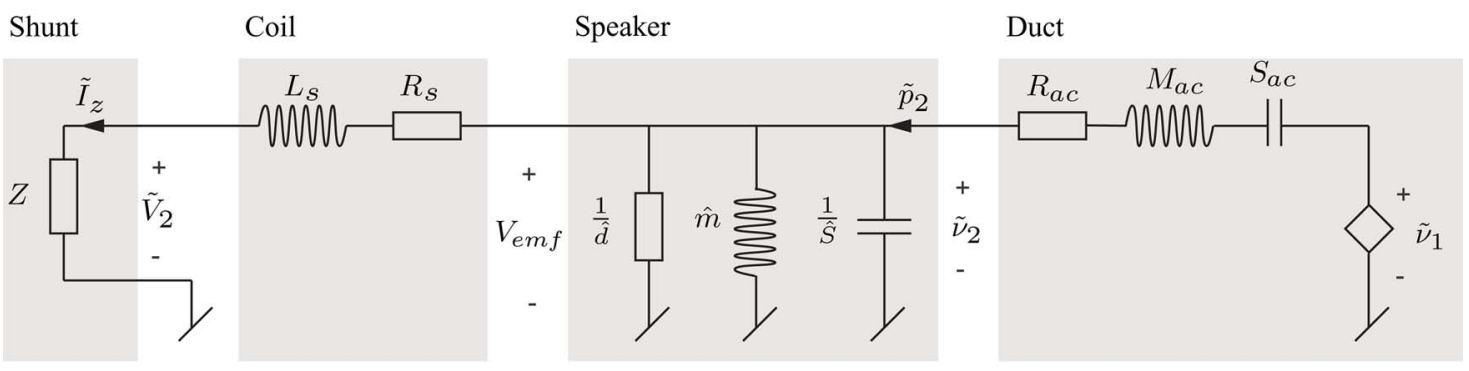

Helmholtz resonator

(b)

Fig. 9. (a) Electrical equivalent model of the composite speaker-enclosure system and (b) simplified electrical equivalent.

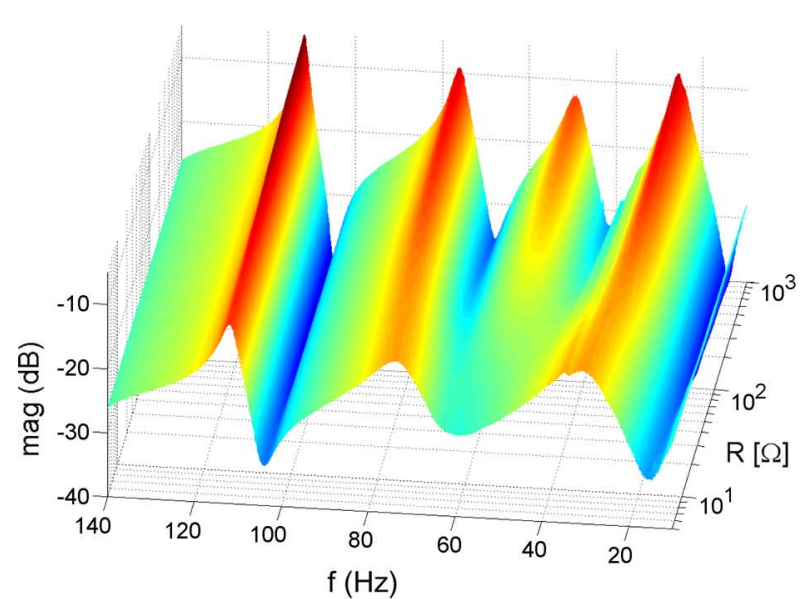

(a)

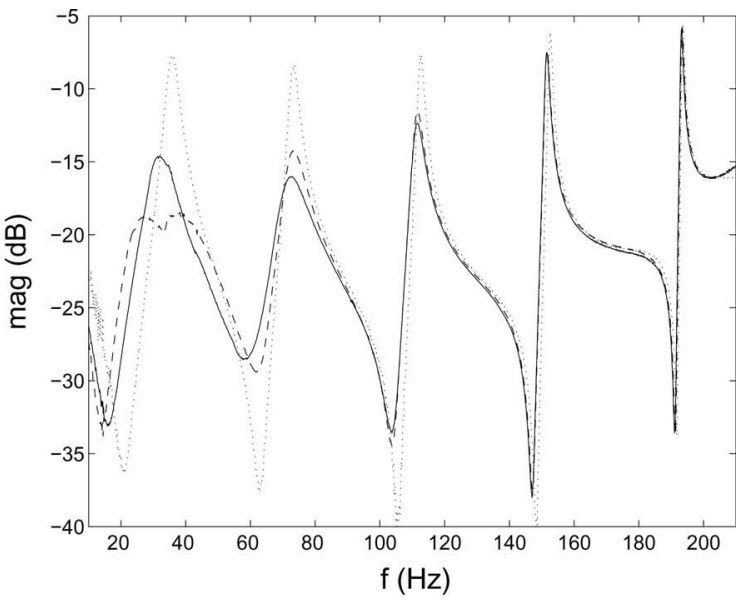

(b)

Fig. 10. Measured magnitude of the transfer-function $G_{p_{1} \nu_{1}}$. (a) Passive $R$ shunt. (b) Passive $R / / C$ shunt, showing duct without speaker (dotted line), duct with open speaker (solid line), and duct with $C / / R$ shunt tuned to the first mode (dashed line).

Experimental results from the application of a $C / / R$ shunt circuit are shown in Fig. 10(b). A capacitance $C=496 \mu \mathrm{F}$, and resistance $R_{p}=8 \Omega$ represents the correct adjustment of resonance frequency and damping. The properly tuned virtual Helmholtz resonator yields a first-mode attenuation of $11 \mathrm{~dB}$. To the knowledge of the authors, a passive capacitor and re- sistor offers the best possible performance commensurate with simplicity and cost. It is important to note that only acoustic modes lower in frequency than both the mechanical and electrical speaker cutoff frequencies can be controlled. Thus, selection of a suitable speaker is critical to the performance of the system. 


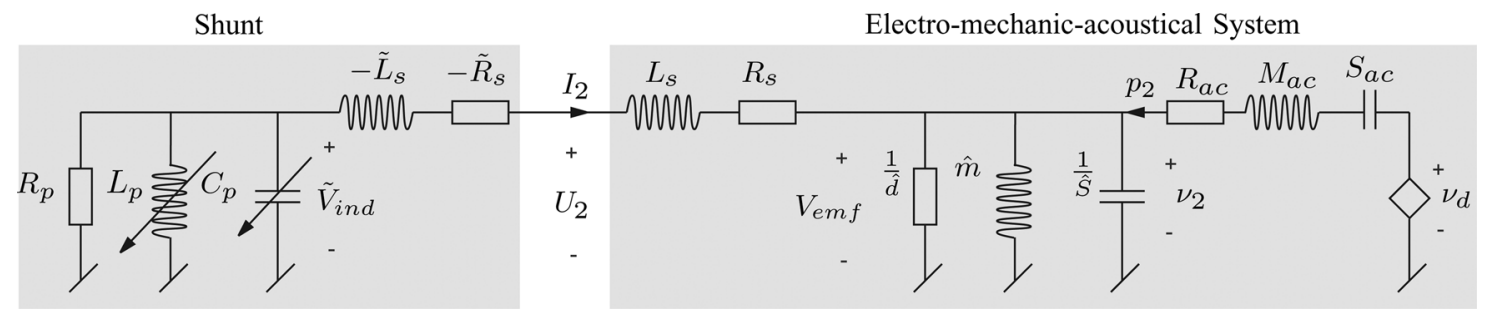

Fig. 11. Shunt with negative $R_{s}-L_{s}$ and parallel $R / / L / / C$ network. All virtual Helmholtz parameters can be varied.

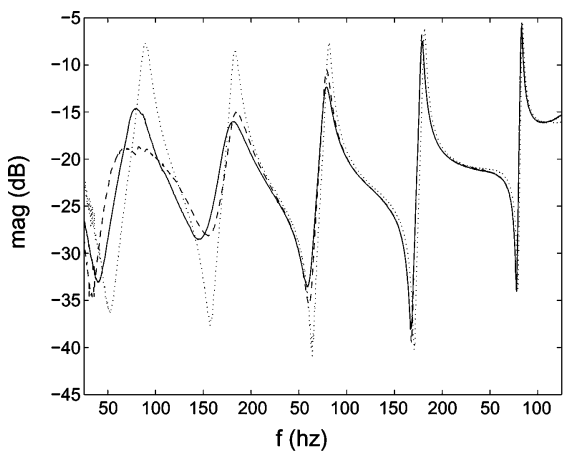

(a)

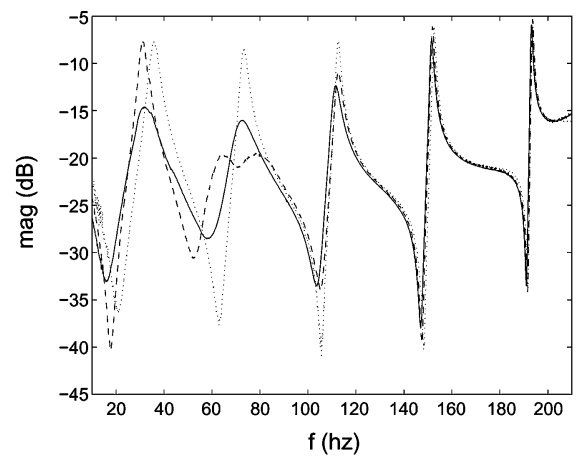

(b)

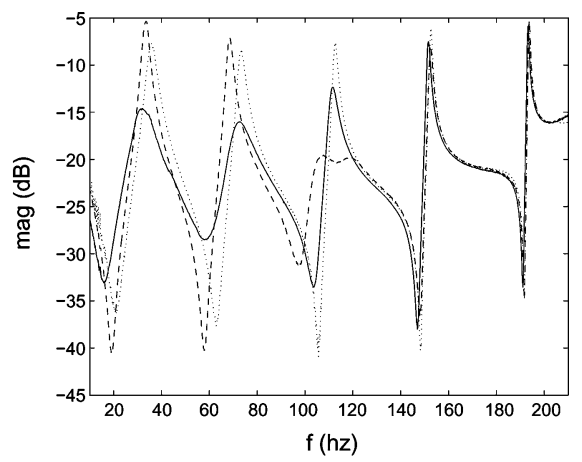

(c)

Fig. 12. Experimental results of the negative $R_{s}-L_{s}$ with $R / / L / / C$ shunt circuit showing the duct response without speaker (dotted line), with open speaker (solid line), and with shunt circuit (dashed line). The shunt circuit is tuned to the (a) first mode, (b) the second mode, and (c) the third mode.

TABLE I

VALUES FOR Negative $R-L$ With $R / / L / / C$ Shunts

\begin{tabular}{|c|c|c|c|c|c|c|}
\hline Mode & $L_{s}[\mathrm{mH}]$ & $R_{s}[\Omega]$ & $R[\Omega]$ & $C[\mu \mathrm{F}]$ & $L[\mathrm{mH}]$ & $f[\mathrm{~Hz}]$ \\
\hline \hline 1 & -12 & -12 & 23 & 116 & $>1000$ & 34 \\
2 & -12 & -12 & 27 & $<1$ & 43.5 & 71 \\
3 & -12 & -12 & 27 & $<1$ & 4.53 & 112 \\
\hline
\end{tabular}

\section{Negative $R-L$ with $R / / L / / C$ Shunts}

By introducing a negative $R-L$ network as illustrated in Fig. 11, a greater authority in the tuning ranges can be achieved. If the negative inductor and resistor are chosen equal, or close to the internal coil impedance, the electrical dynamics of the speaker can be essentially neglected. That is, $\tilde{R}_{s}=-R_{s}$ and $\tilde{L}_{s}=-L_{s}$, where $\tilde{R}_{s}$ and $\tilde{L}_{s}$ are estimates of the internal coil impedance.

With the use of an impedance cancelling network, as shown in Fig. 11, an additional $R_{p} / / L_{p} / / C_{p}$ circuit provides complete control over the virtual Helmholtz resonance frequency. As the capacitance $C_{p}$ appears in parallel with $1 / \hat{S}$, this parameter can be arbitrarily increased with an increase $C_{p}$. The other parameters are independent to variations in $C_{p}$, thus experimental tuning is straight forward. A similar situation occurs with the relationship between $L_{p}$ and $\hat{m}$. This parameter can be varied in either direction to increase or decrease the equivalent resonance frequency. Control over the damping parameter is restricted to values larger than $d$. The total damping is

$$
\hat{d}_{\text {tot }}=\hat{d}+\frac{1}{R_{p}} .
$$

Due to the requirement for negative network elements, practical implementation requires active circuit components. In the following experiments, a synthetic impedance [41], [42] is utilized to implement the shunt circuit impedance. An opampbased negative impedance converter is an alternative for the implementation of negative components.

Fig. 12 demonstrates the experimental application of a negative $\tilde{R}_{s}-\tilde{L}_{s}$ and parallel $R_{p} / / L_{p} / / C_{p}$ for the attenuation of the first, second, and third acoustic modes. The parameters of the shunt circuits are summarized in Table I. Compared to the duct with speaker absent, the acoustic response of each mode is suppressed by between 12 and $14 \mathrm{~dB}$.

1) Online-Tuning: A disadvantage of the previous shunt circuit is that nominal performance is highly sensitive to variations in the acoustic resonance frequencies. In order to provide robustness to such variation, the volume of physical Helmholtz resonators must be mechanically varied with a ball-screw actuator and plunger. Bedout et al. [7] employed a gradient search algorithm to tune the resonance frequency in real time. In this method, the amplitude of a microphone voltage is measured and compared to the former measurement. The resonator volume is adapted to minimize the average measured pressure. Another technique proposed by Kostek and coworkers [9] involves the application of a sine-sweep to identify the correct frequencies of acoustic resonance. After the initial sweep, consecutive sweeps are repeated only after a "significant" increase occurs in the monitored sound pressure. Both methodologies are 


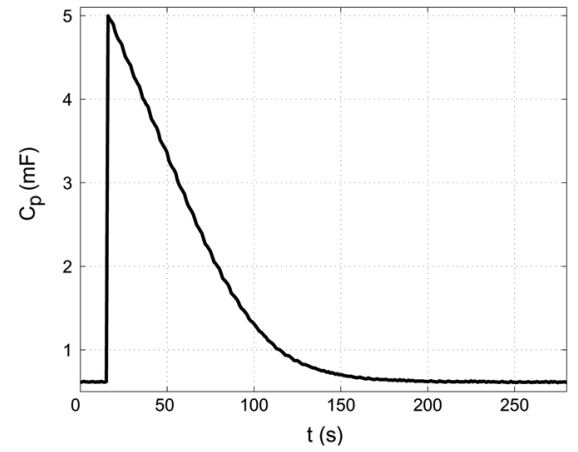

(a)

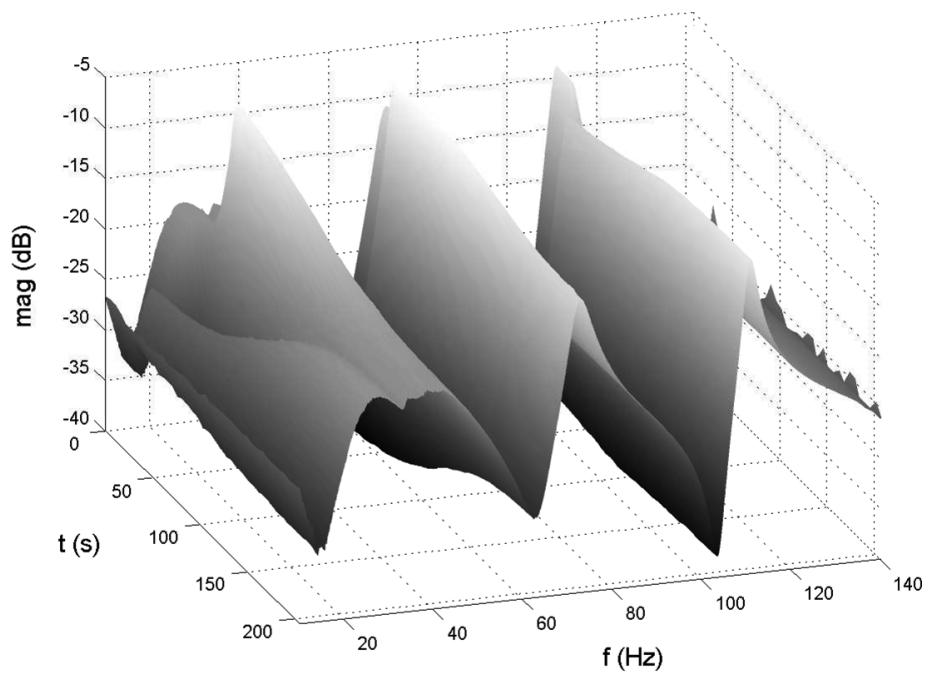

(b)

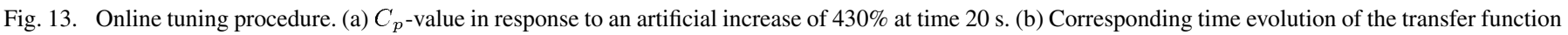
$G_{p_{1} \nu_{1}}$.

reasonably difficult to implement and perform poorly with respect to the speed at which convergence occurs. In addition to implementation difficulties, the requirement for an electric motor and plunger is ungainly, heavy, and prone to failure.

In the field of structural vibration control, techniques for shunt circuit adaptation have been proposed for piezoelectric [43] and electromagnetic [44] transducers. A simple technique based on the phase difference between shunt current and a suitable reference signal provides high adaptation rates and low levels of misadjustment. In the following, this technique is extended to perform adaptation of the virtual Helmholtz resonance frequency.

The technique of relative phase adaptation involves driving the controller resonance frequency to achieve a phase difference of $-\pi / 2$ between a reference signal and the applied or measured current. For the shunted speaker system in Fig. 11, the transferfunction from $p_{2}$ to $\nu_{2}$ can be written in the frequency domain as

$$
\frac{\nu_{2}(s)}{p_{2}(s)}=\frac{s}{s^{2}+s \frac{1}{R C}+\frac{1}{L C}}=\frac{\hat{S} s}{s^{2}+s \hat{d} \hat{S}+\frac{\hat{S}}{\hat{m}}} .
$$

If the integral of $\nu_{2}(s)$, i.e., $\nu_{2}(s) / s$, is taken, the same transferfunction structure used for relative phase adaptation in [43] and [44] is obtained. In this case

$$
\frac{\nu_{2}(s) / s}{p_{2}(s)}=\frac{1 / C}{s^{2}+j \omega \frac{1}{R C}+\frac{1}{L C}}=\frac{\hat{S}}{s^{2}+s \hat{d} \hat{S}+\frac{\hat{S}}{\hat{m}}}
$$

with the phase

$$
\begin{aligned}
\angle \frac{\nu_{2}(s) / s}{p_{2}(s)} & =\Phi \\
& =-\arctan \left(\frac{L / R}{1-L C \omega^{2}}\right) \\
& =-\arctan \left(\frac{\hat{m} \hat{d}}{1-\frac{\hat{m}}{\hat{S}} \omega^{2}}\right)
\end{aligned}
$$

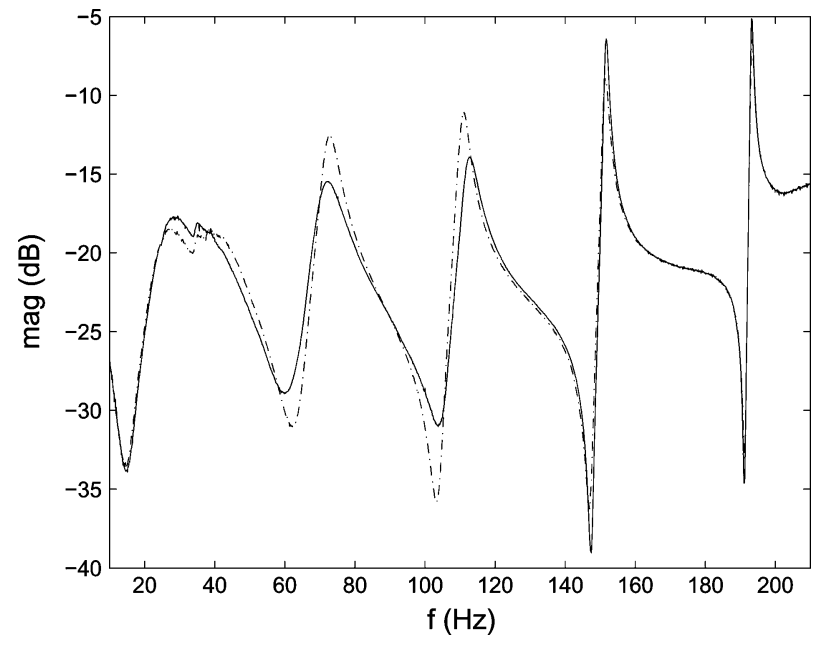

Fig. 14. Measured magnitude of the transfer-function $G_{p_{1} \nu_{1}}$ using negative $\tilde{R}_{s}-\tilde{L}_{s}$ with $R_{p} / / L_{p} / / C_{p}$ and series $L$ shunt to improve damping of higher modes. Negative $R-L$ with $R / / L / / C$ shunt (dash-dot line) and improved shunt with additional series inductor (solid line).

where $s=j \omega$. In light of the structural similarities, relative phase adaptation is applicable using the integrated velocity $\nu_{2}(s) / s$ and pressure $p_{2}(s)$ measured at the speaker baffle. As the induced voltage $V_{\mathrm{emf}}$ is proportional to $\nu_{2}(s)$, the corresponding relative phase adaptation can be written in the time domain as

$$
\frac{\partial L(t)}{\partial t}=\alpha\left[g_{L P}(t) *\left(\int \tilde{V}(t)_{\mathrm{emf}} d t \cdot p_{2}(t)\right)\right]
$$

where $\tilde{V}_{\text {emf }}(t)$ is an estimate of $V_{\text {emf }}(t)$ procured from within the shunt circuit. Here, $g_{L P}(t)$ represents the impulse response of a low-pass filter with a cutoff frequency below $2 \omega_{n}\left(\omega_{n}\right.$ is the resonance frequency to be damped), $\alpha$ the tuning parameter, and $*$ denotes the time domain convolution operator.

An experimental demonstration of online relative phase adaptation can be found in Fig. 13. At time $20 \mathrm{~s}$, a mistuning event occurs where the value of $C_{p}$ is artificially increased by $430 \%$. In Fig. 13(a) and (b), the time evolution of $C_{p}$ and the measured 


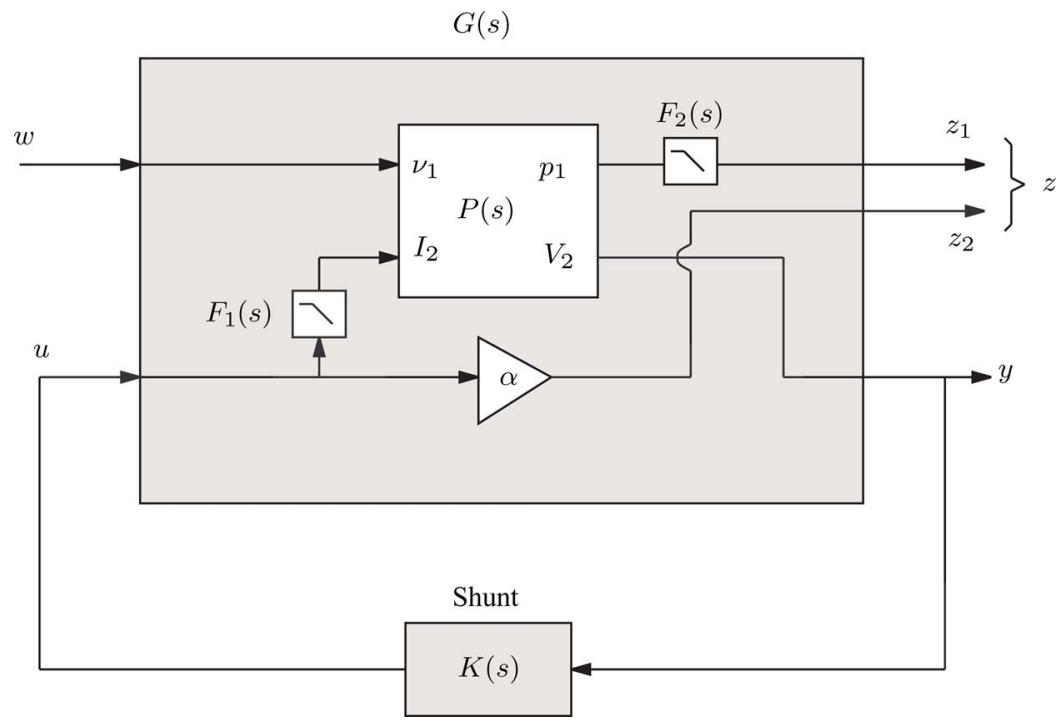

Fig. 15. Block diagram of the composite system with shunted speaker and duct.

magnitude transfer function $G_{p_{1} \nu_{1}}$ are plotted. Even in the presence of such a large disturbance, the shunt circuit is successfully retuned.

2) Improvement With Series L: One of the disadvantages associated with virtual Helmholtz resonators is the narrow-bandwidth of the control action. At resonance frequencies adjacent to that specifically controlled, the response of a lightly damping Helmholtz resonator can be likened to a hard wall, no absorption is provided. As shown in Fig. 12, it may occur that high-frequency acoustic modes are actually enhanced when the speaker is tuned to damp a single mode. This phenomenon can be attributed directly to the reduction of speaker bandwidth with the connection of shunt circuits described in the preceding sections.

The problem can be somewhat alleviated with the addition of a series inductor to the shunt circuit network. By increasing the electrical impedance at high frequencies, the braking behavior of the system can be reduced. As demonstrated in Fig. 14, a series inductor can relax high-frequency speaker stiffening and avoid unwanted enhancement of uncontrolled modes.

\section{Active Shunt Design}

In Section III, electrical shunt impedances are connected across the terminals of an acoustic loudspeaker. With knowledge of the speaker's mechanical and magnetic properties, an electrical impedance can be designed to synthesize the dynamics of a Helmholtz resonator, and hence, augment the damping of resonant acoustic modes. Such techniques are highly intuitive and simple to implement but require detailed knowledge of the loudspeakers physical properties. In practice, the response of the controller must be tuned experimentally. In this section, an alternative approach is taken to electrical impedance design. By casting the acoustic system as a standard multivariable regulator problem, synthesis techniques such as linear quadratic regulator design (LQR), and $\mathcal{H}_{\infty}$ can be applied. Although the resulting impedance does not have a direct physical interpretation, the design process does not require any detailed knowledge of the transducer or acoustic system.

\section{A. Modeling}

Active impedance control of electromagnetic transducers was first studied in [45] and [46]. Essentially the plant is reduced to a set of control inputs $u$, disturbance inputs $w$, measurable outputs $y$, and performance signals $z$. Here, the speaker current $I_{2}$ and the voltage $V_{2}$ are selected as the control variable and measured output, respectively. The control objective remains to attenuate the pressure $p_{1}$ resulting from a velocity disturbance $\nu_{1}$. Note that the control speaker pressure $p_{2}$ is no longer required for design or analysis.

Fig. 15 shows the acoustic system cast as a standard regulator problem. The physical plant $P(s)$ represents the concatenated transducer and acoustic dynamics. Although $P(s)$ can be obtained simply by rearranging the system block diagram shown in Fig. 7, in this paper, system identification is employed to estimate an input-output model. The foremost motivation for the use of system identification is to simplify the design process by removing the need for detailed physical modeling.

The frequency domain subspace class of system identification algorithms [47] has proven useful for the identification of resonant mechanical and acoustic systems [48]. Multivariable frequency domain I/O data from the plant $P(s)$, shown in Fig. 15, is obtained by performing a number of successive single input single output experiments. The response from each input to both outputs is obtained by setting the remaining input to zero and measuring the response of each output successively. Data is recorded using the Polytec vibrometer acquisition software. A total of 533 frequency points were recorded between 0 and $209 \mathrm{~Hz}$. Each frequency response is obtained directly using the ratio of Fourier transforms. No windowing is required, as the excitation is a periodic chirp.

A state-space model of 12 states was identified from the concatenated multivariable frequency data. ${ }^{1} \mathrm{~A}$ good agreement between the identified model and experimental data is shown in Fig. 16. The disturbance $w$ and control signal $u$ are proportional

\footnotetext{
${ }^{1}$ A MATLAB implementation of the subspace algorithm is freely available by contacting the first author.
} 

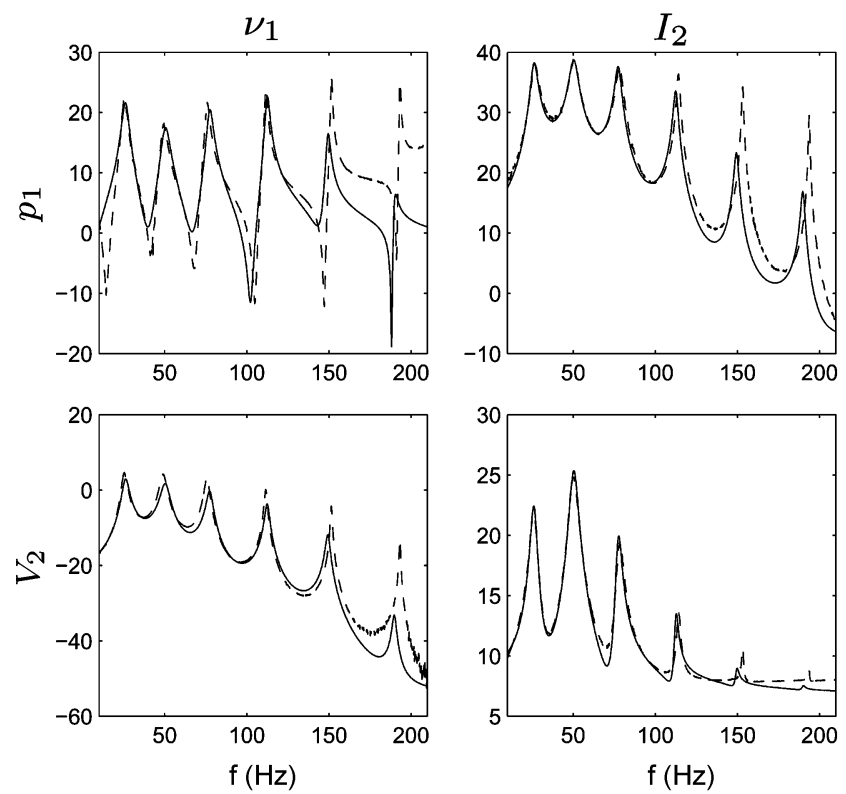

(a)
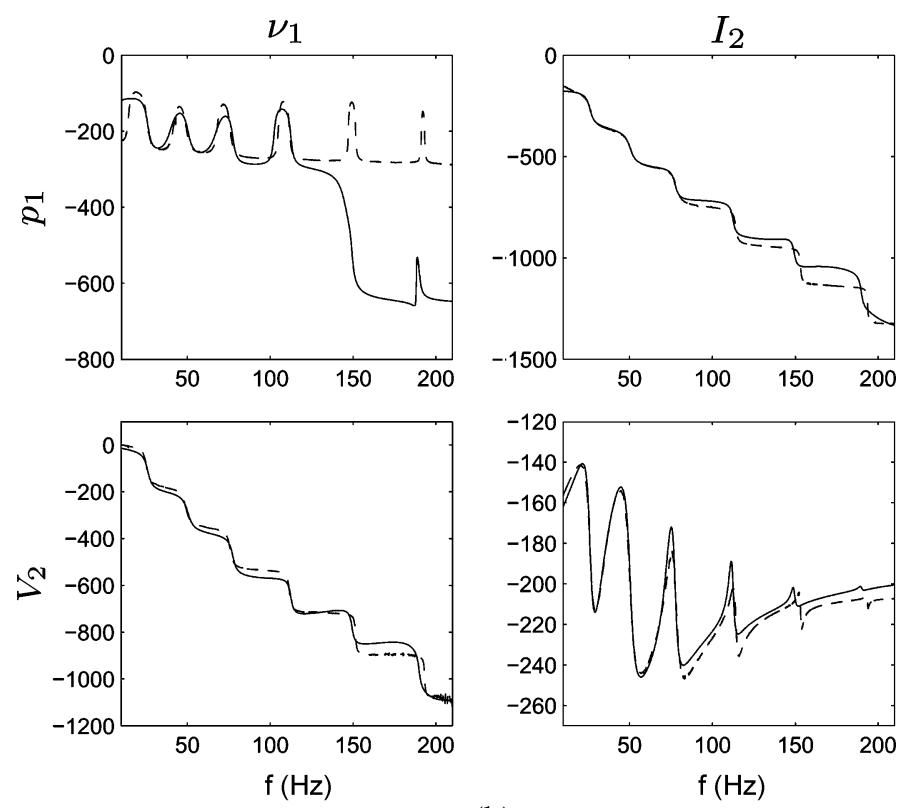

(b)

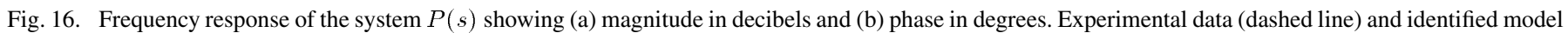
(solid line).

to the disturbance speaker velocity and control speaker current, respectively. Inputs and outputs are measured as the voltages applied to, or measured from the amplifiers and instruments; thus, all system gains are included in the model.

\section{B. Control Design}

Given the $\mathrm{I} / \mathrm{O}$ model $P(s)$, the problem of designing an appropriate impedance can be cast as a standard regulator problem. As shown in Fig. 15, the plant model $P(s)$ is enclosed in another system $G(s)$ for which the regulator $K(s)$ is designed. Aside from the trivial renaming of signals, two low-pass filters have been added. The first-order low-pass filter $F_{1}(s)$ in the control signal path is added to enforce causality in the transfer function from $u$ to $y$. As this transfer function is dominated by the speaker coil impedance, it is naturally improper and has a differentiating action at high frequencies. A second first-order low-pass filter $F_{2}(s)$ is added to the pressure signal $p_{1}$ in order to limit the control bandwidth during the design process. The design objective, reflected by the performance signal $z(s)$, is to minimize the pressure $p_{1}$ subject to a weighting on the required control signal $u$.

As the controller $K(s)$ and low-pass filter $F_{1}(s)$ describe the relationship between speaker coil current and voltage, they can be viewed together as an electrical admittance. Thus, by designing a suitable regulator for the system $G(s)$, we are equivalently designing an electrical admittance for the acoustic system that results in the same performance.

Applying $\mathcal{H}_{\infty}$ control to the problem of admittance synthesis involves finding a controller $K(s)$ that minimizes

$$
J=\left\|F_{L}(G, K)\right\|_{\infty}
$$

where $F_{L}(G, K)$ is the closed-loop transfer function from $w$ to $z$, and the $\mathcal{H}_{\infty}$ norm $\|R(s)\|_{\infty}$ of $R(s)$ is defined as

$$
\|R(s)\|_{\infty}=\max _{\omega} \bar{\sigma}[R(j \omega)]
$$

where $\bar{\sigma}$ denotes the maximum singular value. In the time domain, $\mathcal{H}_{\infty}$ control can be interpreted as minimizing the worstcase induced 2-norm of $z$, i.e.,

$$
J=\inf _{K \in U} \sup _{w \neq 0} \frac{\|z(t)\|_{2}}{\|w(t)\|_{2}}
$$

where $U$ is the set of all stabilizing controllers and $\|r(t)\|_{2}^{2}=$ $\int_{0}^{\infty} r(t)^{\prime} r(t) d t$.

Closely resembling the solution to $\mathcal{H}_{2}$ synthesis, an optimal $\mathcal{H}_{\infty}$ controller can be found through the solution of an algebraic Riccatti equation [49], [50].

Using the $\mu$-synthesis toolbox for MATLAB, an optimal $\mathcal{H}_{\infty}$ regulator $K(s)$ was found that minimizes the specified performance objective. The actual admittance presented to the terminals of the loudspeaker can be found in Fig. 17. Alike the shunt impedances implemented in Section III, the optimal admittance is synthesized artificially as discussed in references [41] and [42]. The admittance influence is illustrated by the simulated open-loop and closed-loop pole locations shown in Fig. 18, a significant amount of damping is added to the first four modes. A gain and phase margin of $3.03 \mathrm{~dB}$ and $14.1^{\circ}$ were measured in the simulated loop-gain. The experimental open-loop and closed-loop frequency response (Fig. 19) shows an attenuation of the first four modes by $9.3,9.5,8.3$, and $8.8 \mathrm{~dB}$, respectively.

The present active shunt circuit has the drawback that its performance and stability are sensitive to model uncertainties, mainly the electromechanical transducer properties. Compared 

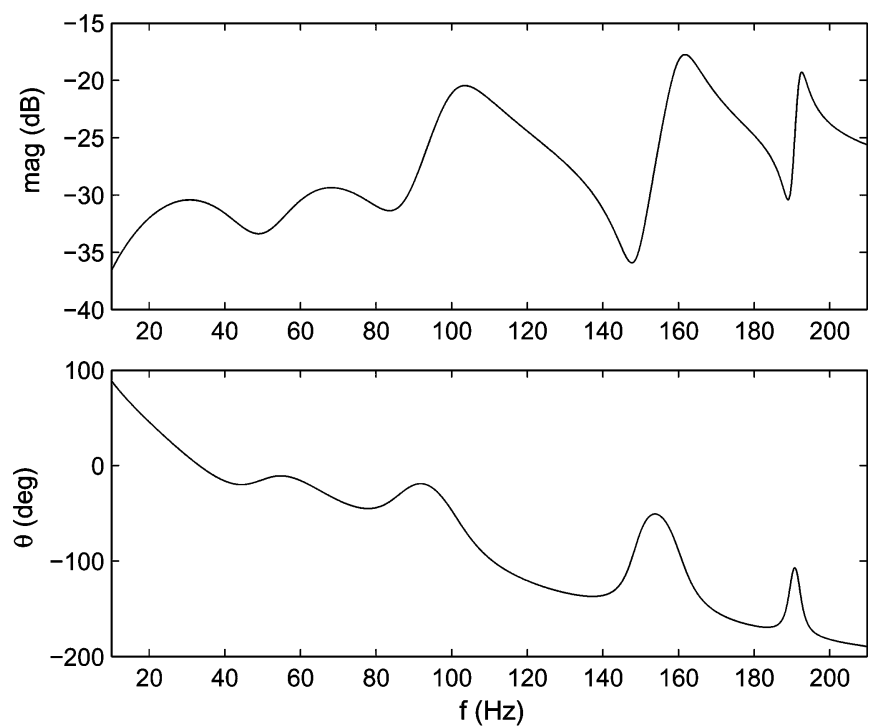

Fig. 17. Actual admittance presented to the speaker coil terminals.

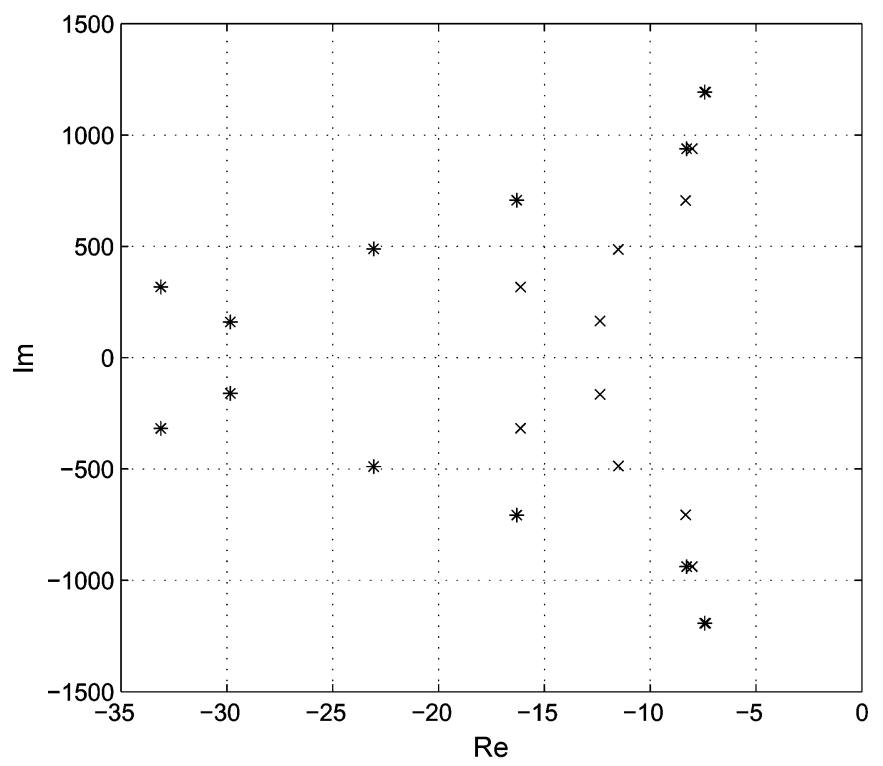

Fig. 18. Open-loop $(\times)$ and closed-loop $(\star)$ pole locations of $G(s)$. The poles of the first four modes are shifted to the left; thus, the damping has been augmented.

to the online-tuned shunt circuit in Section III-C1, no adaptation can be applied to the active shunt circuit. However, a robust control design could be used to improve the robustness.

\section{CONCLUSION}

This paper extends the techniques of passive shunt damping and active shunt control to acoustic loudspeakers. By connecting an electrical impedance to the terminals of a loudspeaker, the mechanical dynamics of the loudspeaker are altered. Based on the mechanical and electrical properties of the loudspeaker, an electrical network can be designed that results in the loudspeaker emulating the acoustic response of a Helmholtz resonator. Highly resonant acoustic modes can be significantly attenuated. In some circumstances, depending on the frequency of resonance and the electromechanical properties of the loudspeaker, a passive capacitor and resistor can be employed to
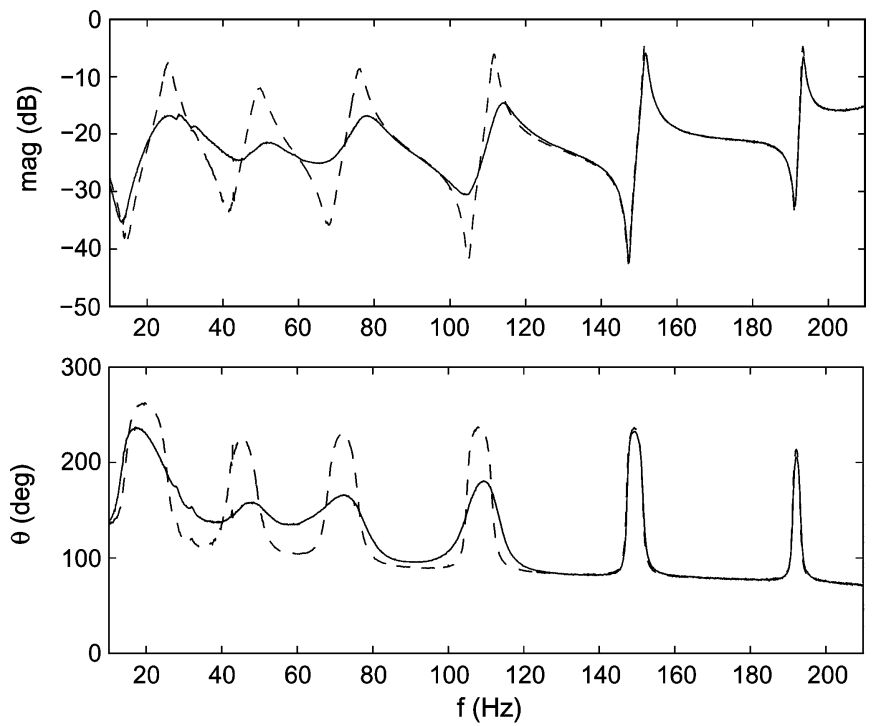

Fig. 19. Open-loop (dashed line) and closed-loop (solid line) magnitude and phase response measured from the applied disturbance velocity $\nu_{1}$ to the resulting pressure $p_{1}$. The first four modes are attenuated by $9.3,9.5,8.3$, and 8.8 $\mathrm{dB}$, respectively.

damp a single acoustic mode. Such simple and inherently stable impedances are useful in applications requiring high reliability and shock resistance, e.g., launch vehicle acoustic control. Experiments performed on a closed acoustic duct demonstrate the effectiveness of the proposed technique.

Due to the narrowband nature of Helmholtz resonators, the resulting acoustic mitigation is highly dependent on the targeted acoustic resonance frequencies. Acoustic systems are typically subject to significant environmental variations, and hence, uncertainty in the frequencies of resonance. A novel technique for online shunt circuit adaptation has been proposed to ensure optimal performance in the presence of environmental variation. Experimental results show independence of damping performance to variation in acoustic resonance frequencies.

By stating the problem of shunt impedance design from a feedback control perspective, standard synthesis techniques such as $L Q R, \mathcal{H}_{\infty}$, and $\mathcal{H}_{2}$ are easily applied to obtain optimal shunt impedances. For the purpose of control design, system identification can be employed to obtain a state-space estimate of the concatenated acoustic and transducer systems. Although impedance designs arising from optimal synthesis do not have an intuitive physical interpretation, more sophisticated performance objectives such as damping of multiple modes are easily considered. Experiments on the acoustic duct system show attenuations of between 8 and $10 \mathrm{~dB}$ for the first four modes.

\section{REFERENCES}

[1] P. Leug, "Process of silencing sound oscillations," U. S. Patent 2043 416, Jun. 9, 1936.

[2] H. F. Olsen and E. G. May, "Electronic sound absorber," J. Acoust. Soc. Amer., vol. 25, pp. 1130-1136, Nov. 1953.

[3] L. L. Beranek, Noise and Vibration Control Engineering: Principles and Applications. New York: Wiley, 1992.

[4] U. Ingard, "On the theory and design of acoustic resonators," J. Acoust. Soc. Amer., vol. 20, pp. 665-682, Nov. 1953.

[5] M. L. Munjal, Acoustics of Ducts and Mufflers. New York: Wiley, 1987. 
[6] R. Chanaud, "Effects of geometry on the resonance frequency of Helmholtz resonators," J. Sound Vib., vol. 178, no. 3, pp. 337-348, 1994.

[7] J. M. D. Bedout, M. A. Franchek, R. J. Bernhard, and L. Mongeau, "Adaptive passive noise control with self-tuning Helmholtz resonators," J. Sound Vib., vol. 202, no. 1, pp. 109-123, 1997.

[8] M. E. Johnson and S. Estève, "Comparison of local and global adaptive strategies for the control of broadband noise in an enclosure using adaptive Helmholtz resonantors," in Proc. Active, 2002, pp. 1111-1120.

[9] T. M. Kostek and M. A. Franchek, "Hybrid noise control in ducts," $J$. Sound Vib., vol. 237, no. 1, pp. 91-100, 2000.

[10] A. Cummings, "The attenuation of sound in unlined ducts with flexible walls," J. Sound Vib., vol. 174, no. 4, pp. 433-450, 1994.

[11] L. Huang, "A theory of reactive control of low-frequency duct noise," J. Sound Vib., vol. 238, no. 4, pp. 575-594, 2000.

[12] R. D. Ford, "Panel sound absorbers," J. Sound Vib., vol. 10, no. 3, pp. 411-423, 1969.

[13] W. Frommhold, H. V. Fuchs, and S. Sheng, "Acoustic performance of membrane absorbers," J. Sound Vib., vol. 170, no. 5, pp. 621-636, 1994.

[14] K. Kido, "Reduction of noise by use of additional sound sources," in Proc. Inter-Noise, 1975, pp. 647-650.

[15] S. M. Kuo and D. R. Morgan, "Active noise control: A tutorial review," Proc. IEEE, vol. 87, no. 6, pp. 943-973, Jun. 1999.

[16] P. A. Nelson and S. J. Elliott, Active Control of Sound. London, U. K.: Academic, 1992.

[17] S. M. Kuo and D. R. Morgan, Active Noise Control Systems. New York: Wiley, 1996.

[18] C. P. Nowicki, D. P. Mendat, and D. G. Smith, "Active attenuation of motor/blower noise," in Proc. Noise-Con., 1994, pp. 415-420.

[19] U. Emborg, "Application of active noise control in Saab 340 and Saab 2000," presented at the Nordic Conf. Veh. Mach. Vib., Stockholm, Sweden, 1994.

[20] C. R. Fuller and A. H. von Flotow, "Active control of sound and vibration,” IEEE Control Syst. Mag., vol. 15, no. 6, pp. 9-19, Dec. 1995.

[21] S. O. R. Moheimani, H. R. Pota, and I. R. Petersen, "Active control of noise and vibration in acoustic ducts and flexible structures-A spatial control approach," in Proc. Amer. Control Conf., 1998, pp. 2601-2605.

[22] A. J. Hull, C. J. Radcliffe, and S. C. Southward, "Global active noise control of a one-dimensional acoustic duct using a feedback controller," Trans. ASME, vol. 115, pp. 488-494, Sep. 1993.

[23] R. L. Clark and D. G. Cole, "Active damping of enclosed sound fields through direct rate feedback control," J. Acoust. Soc. Amer., vol. 93, no. 3, pp. 1710-1716, Mar. 1995.

[24] J. Hong, J. C. Akers, R. Venugopal, M. Lee, A. G. Sparks, P. D. Washabaugh, and D. Bernstein, "Modeling, identification, and feedback control of noise in an acoustic enclosure," IEEE Trans. Control Syst. Technol., vol. 4, no. 3, pp. 283-291, May 1996.

[25] S. A. Lane and R. L. Clark, "Dissipative feedback control of a reverberant enclosure using a constant volume velocity source," ASME J. Vib. Acoust., vol. 120, pp. 987-993, Oct. 1998.

[26] — , "Improving loudspeaker performance for active noise control," J. Audio Eng. Soc., vol. 46, no. 6, pp. 508-519, Jun. 1998.

[27] Y. Li and G. T.-C. Chiu, "Control of loudspeakers using disturbanceobserver-type velocity estimation," IEEE/ASME Trans. Mechatronics, vol. 10, no. 1, pp. 111-117, Feb. 2005.

[28] D. J. Leo and D. Limpert, "A self-sensing technique for active acoustic attenuation," J. Sound Vib., vol. 235, no. 5, pp. 863-873, 2000

[29] R. L. Clark and K. D. Frampton, "Phase compensation for feedback control of enclosed sound fields," J. Sound Vib., vol. 195, no. 5, pp. 701-718, 1996.

[30] D. Guicking and K. Karcher, "Active impedance control for one-dimensional sound," ASME J. Vib., Acoust., Stress, Reliab. Des., vol. 106, pp. 393-396, 1984.

[31] D. Guicking and E. Lorentz, "An active sound absorber with porous plate," ASME J. Vib., Acoust., Stress, Reliab. Des., vol. 106, pp. 389-392, 1984.

[32] M. Furstoss, D. Thenail, and M. A. Galland, "Surface impedance control for sound absorption: Direct and hybrid passive/active strategies," J. Sound Vib., vol. 203, no. 2, pp. 219-236, 1997.

[33] O. Lacour, M. A. Galland, and D. Thenail, "Preliminary experiments on noise reduction in cavaties using active impedance changes," J. Sound Vib., vol. 230, no. 1, pp. 69-99, 2000.

[34] J. P. Smith, B. D. Johnson, and R. A. Burdisso, "A broadband passiveactive sound absorption system," J. Acoust. Soc. Amer., vol. 106, no. 5, pp. 2646-2652, November 1999.
[35] T. Samejima, "A state feedback electro-acoustic transducer for active control of acoustic impedance," J. Acoust. Soc. Amer., vol. 113, no. 3, pp. 1483-1491, 2003.

[36] J. D. Kemp and R. L. Clark, "Noise reduction in a launch vehicle fairing using actively tuned loudspeakers," J. Acoust. Soc. Amer., vol. 113, no. 4, pp. 1986-1994, Apr. 2003.

[37] B. J. Zimmer, S. P. Lipshitz, K. A. Morris, J. Vanderkooy, and E. E. Obasi, "An improved acoustic model for active noise control in a duct," Trans. ASME, vol. 125, pp. 382-395, Sep. 2003.

[38] H. R. Pota and A. G. Kelkar, "Modeling and control of acoustic ducts," J. Vib. Acoust., vol. 123, pp. 2-10, Jan. 2001.

[39] N. W. Hagood and A. Von Flotow, "Damping of structural vibrations with piezoelectric materials and passive electrical networks," J. Sound Vib., vol. 146, no. 2, pp. 243-268, 1991.

[40] S. Moheimani, "A survey of recent innovations in vibration damping and control using shunted piezoelectric transducers," IEEE Trans. Control Syst. Technol., vol. 11, no. 4, pp. 482-494, Jul. 2003.

[41] A. J. Fleming, S. O. R. Moheimani, and S. Behrens, "Synthesis and implementation of sensor-less active shunt controllers for electromagnetically actuated systems," IEEE Trans. Control Syst. Technol., vol. 13, no. 2, pp. 246-261, Mar. 2005.

[42] A. J. Fleming, S. Behrens, and S. O. R. Moheimani, "Synthetic impedance for implementation of piezoelectric shunt-damping circuits," IEE Electron. Lett., vol. 36, no. 18, pp. 1525-1526, Aug. 2000.

[43] D. Niederberger, A. J. Fleming, S. O. R. Moheimani, and M. Morari, "Adaptive multi-mode resonant piezoelectric shunt damping," J. Smart Mater. Structures, IOP Publishing, vol. 13, pp. 1025-1035, Jul. 2004.

[44] D. Niederberger, S. Behrens, A. J. Fleming, S. O. R. Moheimani, and M. Morari, "Adaptive electromagnetic shunt damping," IEEE/ASME Trans. Mechatronics, vol. 11, no. 1, pp. 103-108, Feb. 2006.

[45] A. Fleming, S. O. R. Moheimani, and S. Behrens, "Synthesis and implementation of sensor-less active shunt controllers for electromagnetically actuated systems," IEEE Trans. Control Syst. Technol., vol. 13, no. 2, pp. 246-261, Mar. 2005.

[46] C. K. Paulitsch, P. Gardonio, and S. J. Elliott, "Active vibration damping using a self-sensing electrodynamic actuator," in Proc. SPIE Smart Mater. Structures, Damping Isolation, 2004, pp. 282-293.

[47] T. McKelvey, H. Akcay, and L. Ljung, "Subspace based multivariable system identification from frequency response data," IEEE Trans. Autom. Control, vol. 41, no. 7, pp. 960-978, Jul. 1996.

[48] T. McKelvey, A. J. Fleming, and S. O. R. Moheimani, "Subspace based system identification for an acoustic enclosure," ASME J. Vib. Acoust., vol. 124, no. 3, pp. 414-419, Jul. 2002.

[49] J. C. Doyle, K. Glover, P. Khargonekar, and B. Francis, "State-space solutions to standard $\mathcal{H}_{2}$ and $\mathcal{H}_{\infty}$ problems," IEEE Trans. Autom. Control, vol. 34, no. 8, pp. 831-847, Aug. 1989.

[50] J. C. Doyle, B. A. Francis, and A. R. Tannenbaum, Feedback Control Theory. New York: Macmillan, 1992.

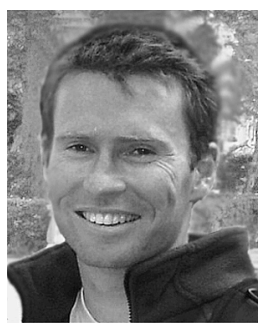

Andrew J. Fleming (M'03) was born in Dingwall, Scotland, in 1977. He received the B.S. degree in electrical engineering and the Ph.D. degree from The University of Newcastle, Callaghan, Australia, in 2000 and 2004, respectively.

He is presently an Australian Research Council APD Fellow stationed at the School of Electrical Engineering and Computer Science, The University of Newcastle. In 2005, he was a Research Academic at the ARC Centre for Complex Dynamic Systems and Control, The University of Newcastle. His research includes microcantilever sensors, nanopositioning, and sensorless control of sound and vibration.

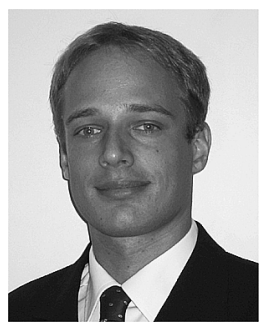

Dominik Niederberger was born in Switzerland in 1976. He received the diploma and the Ph.D. degree from ETH Zurich, Zurich, Switzerland, in 2001 and 2005, respectively.

Since 2006, he has been a Research and Development Engineer at Sensirion AG, Staefa, Switzerland. His research interests include vibration and noise control, model predictive control, hybrid systems and sensor technology.

Dr. Niederberger was a recipient of the ETH Medal from ETH Zurich for his Ph.D. dissertation "Smart damping materials using shunt control," in 2005. 


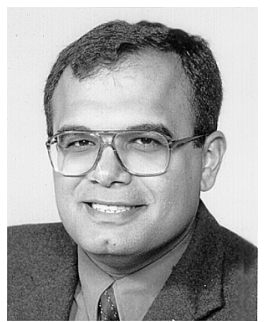

S. O. Reza Moheimani (SM'00) received the Ph.D. degree in electrical and electronic engineering from the University of New South Wales at Australian Defence Force Academy, Australia, in 1996.

Following a research position at the University of New South Wales, he joined the University of Newcastle, Callaghan, Australia, in 1997, where he is currently an Associate Professor in the School of Electrical Engineering and Computer Science, the Director of the Laboratory for Dynamics and Control of Smart Structures, and a Associate Director of the ARC Centre for Complex Dynamic Systems and Control, an Australian Government Centre of Excellence. He has published two books, edited several volumes, and over 150 articles in areas of robust control and estimation, smart structures, active noise and vibration control, mechatronic systems, and nanotechnology.

Dr. Moheimani is an Associate Editor of several international journals including the IEEE TRANSACTIONS ON CONTROL Systems TEChNOLOGY, and has chaired a number of international workshops and conferences.

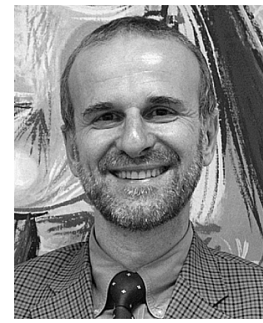

Manfred Morari (F'05) received the diploma from ETH Zurich, Zurich, Switzerland, in 1974 and the Ph.D. degree from the University of Minnesota, Minneapolis, both in chemical engineering, in 1977.

$\mathrm{He}$ was the McCollum-Corcoran Professor of Chemical Engineering and Executive Officer for Control and Dynamical Systems at the California Institute of Technology, Pasadena. In 1994, he was appointed head of the Automatic Control Laboratory at ETH Zurich. His research interests include hybrid systems and the control of biomedical systems. He was elected to the National Academy of Engineering (United States). He has held appointments with Exxon and ICI plc and serves on the technical advisory board of several major corporations.

Prof. Morari is a recipient of numerous awards, among them the Donald P. Eckman Award of the Automatic Control Council, the Allan P. Colburn Award, the Professional Progress Award of the AIChE, the Curtis W. McGraw Research Award of the ASEE, Doctor Honoris Causa from Babes-Bolyai University, and the IEEE Control Systems Technical Field Award. 